\title{
ASTHMA AND INFLAMMATION
}

Dr Adrian Chan Kwok Wai

\section{ABSTRACT}

Asthma is a chronic inflammatory airway disease, for which the cornerstone of asthma therapy is inhaled corticosteroids. However, long term clinical outcomes are variable, and not all patients respond optimally to corticosteroids. Underpinning this observation is that asthma is a heterogeneous disease consisting of phenotypes that are driven by different inflammatory pathways. In this article, we will discuss the different inflammatory mechanisms of asthma to better define patient characteristics and help improve patient outcomes with newer specific-targeted asthma therapies.

\section{Keywords: asthma, endotypes, type 2 inflammation}

\section{SFP2019; 45(6) : 5-6}

\section{INTRODUCTION}

The Global Initiative for Asthma (GINA) defines asthma as a syndrome characterised by variable expiratory airflow limitation, airway hyper-responsiveness and chronic airway inflammation. ${ }^{1}$ Poorly controlled or severe asthma places a significant burden on clinical outcomes and utilisation of healthcare resources. Although effective therapy in the form of inhaled corticosteroids is widely available, it is increasingly recognised as a "one size fits all" treatment approach that does not work for asthma, with a significant proportion of asthma patients not responding optimally to corticosteroids, even after accounting for nonadherence to treatment and asthma triggers. A key reason is that certain inflammatory types are steroid resistant. Thus, there is a recent emphasis on determining the inflammatory mechanisms of asthma, known as endotyping, to accurately predict disease outcomes and tailor appropriate therapies for each individual patient. $^{2}$

Previously, one approach to classify asthma was based on the atopic or allergic status of a patient. ${ }^{3}$ With greater understanding of asthma inflammation, asthma has been broadly classified as possessing type 2 (T2) high or T2-low inflammation, based on the dominant CD4+ T-cell response. More recently, in severe corticosteroid-dependent asthmatics, four patterns of cellular inflammation have been reported in the airways: eosinophilic, neutrophilic, and mixed inflammatory (neutrophils and eosinophils both increased) and pauci-granulocytic (eosinophils and neutrophils not elevated). ${ }^{4}$ In the following sections, we will describe in more detail the various types of inflammation and their impact on disease outcomes and therapy.

\section{Dr Adrian Chan Kwok Wai}

Consultant

Respiratory \& Critical Care Medicine

Singapore General Hospital

\section{ASTHMA AND T2-HIGH INFLAMMATION}

T2-high asthma is currently the best understood inflammatory endotype. In response to exposure to a trigger (e.g. allergens, viruses, pollutants), antigen-presenting cells induce a T-helper cell type 2 (Th2) differentiation. Th2 cells secrete cytokines such as interleukins 4, 5 and 13 (IL-4, IL-5 and IL-13)which drive the downstream recruitment of effector cells (mast cells, eosinophils, basophils) and facilitate the isotype switching of $\mathrm{B}$ cells-secreted immunoglobulins to Immunoglobulin E (IgE). IL-4 and IL-13 exert direct effects on the airway by promoting subepithelial fibrosis and airway remodelling, the latter also increases goblet cell hyperplasia, mucus hypersecretion and airway hyper-reactivity. ${ }^{6}$ Another immune cell, known as the type 2 innate lymphoid cells (ILC2s), was recently discovered to propagate similar immune responses by producing IL-5 and IL13. ${ }^{7}$ Thus, T2-high asthma is characterized by increased levels of eosinophils, exhaled fraction of nitric oxide (a marker of Il-13 driven inflammation in the airway), serum Immunoglobulin $\mathrm{E}$ (IgE) and type 2 cytokines in the blood and/or airway.

Relating to clinical phenotypes, T2-high inflammation has been described in several groups:

a) early-onset allergic asthma, seen in younger patients and is associated with positive skin allergy tests or serum specific $\operatorname{IgE}$,

b) late-onset eosinophilic asthma, seen in older patients without evidence of atopy and is surprisingly steroid-resistant.

c) aspirin-exacerbated respiratory disease (AERD) arising from dysregulated arachidonic acid (AA) metabolism and cysteinyl leukotrienes production, which produces a clinical syndrome of severe asthma and nasal polyposis.

\section{ASTHMA AND T2-LOW INFLAMMATION}

Up to 50 percent of patients may not possess eosinophilic airway inflammation, and in severe asthmatics, about 63 percent of patients do not possess Th- 2 high inflammation. ${ }^{8,9}$ These patients may be termed as having T2-low asthma. In contrast to T2-high asthma, T2-low asthma is less understood and is likely to involve different immune mechanisms. One hypothesis is a distinct inflammation driven by a different class of $\mathrm{T}$ lymphocytes known as Th17 cells, which secrete cytokines such as IL-17. IL17 has been demonstrated in animal models to increase airway hyper-responsiveness, and is also associated with neutrophilic inflammation and corticosteroid resistance. ${ }^{10}$ Patients with T2low asthma are more likely to have an adult-onset disease with no prior childhood allergy features. ${ }^{11}$

In pauci-granulocytic asthma, the primary pathogenetic mechanism is likely to be airway smooth muscle dysfunction that induces airway hyper-responsiveness. ${ }^{12}$ 


\section{EVALUATION AND MANAGEMENT}

Evaluating asthma inflammatory mechanisms using biomarkers is important to distinguish between different asthma phenotypes and identify patients suitable for specific-targeted therapies. Currently available investigations for allergic or eosinophilic asthma include FENO, peripheral blood eosinophilia and determining the inflammatory cellular profile of induced sputum and/or bronchioalveolar lavage. However, to date, specific biomarkers of type 2-low asthma have not been described.

T2-high asthma appears to be responsive to steroid therapy. Selected T2-high asthmatics may have relative steroid resistance and may be candidates for add-on biologic therapy. Currently, anti-IgE and anti-IL-5 are available in Singapore. Patients with neutrophilic asthma tend to be resistant to corticosteroids and may benefit from other forms of anti-inflammatory therapy, such as long-term macrolide therapy. ${ }^{13}$ Despite the severity of their disease, currently, there are no proven effective treatment options for patients with mixed inflammatory asthma. Asthmatics with pauci-granulocytic inflammation are less likely to respond to anti-inflammatory therapy. ${ }^{12}$ They may instead benefit from therapies targeting smooth muscle dysfunction such as bronchial thermoplasty, a bronchoscopic procedure that delivers controlled thermal energy to the airway wall to reduce airway smooth muscle bulk. ${ }^{14}$

\section{REFERENCES}

1 Global Initiative for Asthma. Global Strategy for Asthma Management and Prevention, 2019. [Accessed 1 August 2019]. Available from: https://ginasthma.org/wp-content/uploads /2019/06/GINA-2019-main-report-June-2019-wms.pdf

2 Kuruvilla ME, Lee FE, Lee GB. Understanding asthma phenotypes, endotypes, and mechanisms of disease. Clinical reviews in allergy \& immunology. 2019 Apr 15;56(2):21933.doi:10.1007/s12016-018-8712-13

3 Johansson SG, Bieber T, Dahl R, Friedmann PS, Lanier BQ, Lockey RF, Motala C Martell JA, Platts-Mills TA, Ring J, Thien F. Revised nomenclature for allergy for global use: Report of the Nomenclature Review Committee of the World Allergy Organization, October 2003. Journal of allergy and clinical immunology. 2004 May 1;113(5):832-6. doi:10.1016/j. jaci.2003.12.591

4 Wenzel SE, Schwartz LB, Langmack EL, Halliday JL, Trudeau JB, Gibbs RL, Chu HW. Evidence that severe asthma can be divided pathologically into two inflammatory subtype with distinct physiologic and clinical characteristics. American journal of respiratory and critical care medicine. 1999 Sep 1;160(3):1001-8. doi:10.1164/ajrccm.160.3.9812110

5 Gibeon D, Menzies-Gow AN. Targeting interleukins to treat severe asthma. Expert review of respiratory medicine. 2012 Aug 1;6(4):423-39. doi:10.1586/ers.12.38

6 Hershey GK. IL-13 receptors and signaling pathways: an evolving web. Journal of Allergy and Clinical Immunology. 2003 Apr 1;111(4):677-90. doi:10.1067/mai.2003.1333

7 Moro K, Yamada T, Tanabe M, Takeuchi T, Ikawa T, Kawamoto H, Furusawa JI, Ohtani $\mathrm{M}$, Fujii $\mathrm{H}$, Koyasu S. Innate production of $\mathrm{T} \mathrm{H} 2$ cytokines by adipose tissue-associated c-Kit+ Sca-1+ lymphoid cells. Nature. 2010 Jan;463(7280):540-4 doi:10.1038/nature08636

8 Green RH, Brightling CE, Woltmann G, Parker D, Wardlaw AJ, Pavord ID. Analysis of induced sputum in adults with asthma: identification of subgroup with isolated sputum neutrophilia and poor response to inhaled corticosteroids. Thorax. 2002 Oct 1;57(10):875-9. doi:10.1136/thorax. 57.10 .875

9 Pavlidis S, Loza M, Baribaud F, Kuo CH, Rowe A, Lutter R, Hoda U, Rossios C, Sousa A, Corfield J, Adcock I. Th2 subsetting of U-BIOPRED asthma subjects based on airway transciptomic profiles.doi:10.1183/13993003.congress-2015.OA1772

10 McKinley L, Alcorn JF, Peterson A, DuPont RB, Kapadia S, Logar A, Henry A, Irvin CG, Piganelli JD, Ray A, Kolls JK. TH17 cells mediate steroid-resistant airway inflammation and airway hyperresponsiveness in mice. The Journal of Immunology. 2008 Sep 15;181(6):408997.doi:10.4049/jimmunol.181.6.4089

11 Martin PE, Matheson MC, Gurrin L, Burgess JA, Osborne N, Lowe AJ, Morrison S, Mészáros D, Giles GG, Abramson MJ, Walters EH. Childhood eczema and rhinitis predict atopic but not nonatopic adult asthma: a prospective cohort study over 4 decades. Journal of Allergy and Clinical Immunology. 2011 Jun 1;127(6):1473-9. doi:10.1016/j.jaci.2011.02.041

12 Svenningsen S, Nair P. Asthma endotypes and an overview of targeted therapy for asthma. Frontiers in medicine. 2017 Sep 26;4:158. doi:10.3389/fmed.2017.00158

13 Gibson PG, Yang IA, Upham JW, Reynolds PN, Hodge S, James AL, Jenkins C, Peter MJ, Marks GB, Baraket M, Powell H. Effect of azithromycin on asthma exacerbations and quality of life in adults with persistent uncontrolled asthma (AMAZES): a randomised, double-blind, placebo-controlled trial. The Lancet. 2017 Aug 12;390(10095):659-68. doi:10.1016/s0140-6736(17)31281-3

14 Cox C, Kjarsgaard M, Surette MG, Cox PG, Nair P. A multidimensional approach to the management of severe asthma: Inflammometry, molecular microbiology and bronchial thermoplasty. Canadian respiratory journal. 2015;22(4):221-4. doi:10.1155/2015/459187

\section{LEARNING POINTS}

- Asthma is a heterogeneous disease characterised by different inflammatory mechanisms.

- Asthma can be broadly classified into T2-high asthma and T2-low asthma. However, with a greater understanding of biologic pathways and the immune cells involved, the aim is to accurately characterise asthma characteristics and lead to targeted therapeutic approaches.

- Patients with T2-high asthma are more likely to be corticosteroid responsive and may benefit from newer biologic therapy. In contrast, T2-low asthma tends to demonstrate corticosteroid resistance. 